\title{
The Effect of Aliphatic Carboxylic Acids on Olfaction-Based Host-Seeking of the Malaria Mosquito Anopheles gambiae
} sensu stricto

\author{
Renate C. Smallegange • Yu Tong Qiu • \\ Gabriella Bukovinszkiné-Kiss • Joop J. A. Van Loon • \\ Willem Takken
}

Received: 16 February 2009/Revised: 20 May 2009 / Accepted: 5 June 2009 /Published online: 23 July 2009

(C) The Author(s) 2009. This article is published with open access at Springerlink.com

\begin{abstract}
The role of aliphatic carboxylic acids in hostseeking response of the malaria mosquito Anopheles gambiae sensu stricto was examined both in a dual-choice olfactometer and with indoor traps. A basic attractive blend of ammonia + lactic acid served as internal standard odor. Single carboxylic acids were tested in a tripartite blend with ammonia + lactic acid. Four different airflow stream rates $(0.5,5,50$, and $100 \mathrm{ml} / \mathrm{min})$ carrying the compounds were tested for their effect on trap entry response in the olfactometer. In the olfactometer, propanoic acid, butanoic acid, 3-methylbutanoic acid, pentanoic acid, heptanoic acid, octanoic acid, and tetradecanoic acid increased attraction relative to the basic blend. While several carboxylic acids were attractive only at one or two flow rates, tetradecanoic acid was attractive at all flow rates tested. Heptanoic acid was attractive at the lowest flow rate $(0.5 \mathrm{ml} / \mathrm{min})$, but repellent at 5 and $50 \mathrm{ml} / \mathrm{min}$. Mixing the air stream laden with these 7 carboxylic acids together with the headspace of the basic blend increased attraction in two quantitative compositions. Subtraction of single acids from the most attractive blend revealed that 3-methylbutanoic acid had a negative effect on trap entry response. In the absence of tetradecanoic acid, the blend was repellent. In assays with MM-X traps, both a blend of 7 carboxylic acids + ammonia + lactic acid (all applied from low density polyethylene-sachets)
\end{abstract}

A patent has been filed for Blends A and B listed in Table 5, under number European Patent number 09154467.6.

Electronic supplementary material The online version of this article (doi:10.1007/s10886-009-9668-7) contains supplementary material, which is available to authorized users.

R. C. Smallegange $(\bowtie) \cdot$ Y. T. Qiu • G. Bukovinszkiné-Kiss •

J. J. A. Van Loon - W. Takken

Laboratory of Entomology, Wageningen University,

PO Box 8031, 6700 EH Wageningen, The Netherlands

e-mail: renate.smallegange@wur.nl and a simple blend of ammonia + lactic acid + tetradecanoic acid were attractive. The results show that carboxylic acids play an essential role in the host-seeking behavior of An. gambiae, and that the contribution to blend attractiveness depends on the specific compound studied.

Keywords Anopheles gambiae sensu stricto. Diptera: Culicidae · Mosquito · Olfactometer · Host-seeking behavior $\cdot$ Human odor Attraction $\cdot$ Carboxylic acids

\section{Introduction}

Several anthropophilic mosquito species are attracted to volatiles from human skin (Schreck et al. 1981; De Jong and Knols 1995, 1996; Geier et al. 1996; Mboera et al. 1998). Although it is assumed that several chemical compounds are involved in this behavior, their identities remain poorly understood. More than 300 volatile compounds have been identified from human skin (Bernier et al. 2000; Curran et al. 2005; Penn et al. 2007; Gallagher et al. 2008).

Lactic acid is an attractant of the yellow fever mosquito Aedes aegypti L. (Acree et al. 1968; Smith et al. 1970; Eiras and Jepson 1991; Geier et al. 1996; Bernier et al. 2002). Knols et al. (1997) demonstrated that a blend of 12 carboxylic acids affected the behavior of the malaria vector Anopheles gambiae Giles sensu stricto. In previous studies, we found that An. gambiae females are attracted to ammonia alone (Braks et al. 2001; Smallegange et al. 2005). The attractiveness of ammonia was not increased by lactic acid. However, a synergistic effect was found for the combination of ammonia, lactic acid, and a blend of carboxylic acids, even though the latter was found to be repellent on its own. Lactic acid proved essential for the synergism (Smallegange 
et al. 2005). Carboxylic acids also play a crucial role in the behavior of Ae. aegypti (Bosch et al. 2000) together with ammonia and lactic acid (Geier et al. 1999). Humans have uniquely high levels of skin-borne lactic acid compared to other mammals and chickens. Therefore, lactic acid may play a critical role in the host-seeking behavior of anthropophilic mosquitoes, which seem to have adapted to utilize this human compound (Dekker et al. 2002). By contrast, ammonia is a typical animal excretory product found on the skin of all vertebrates. Skin bacteria convert human skin lipids into unusual long chain carboxylic acids, whereas long chain carboxylic acids can be converted into volatile carboxylic acids (C2-C14). In addition, short chain carboxylic acids (C2-C5) can be derived from glycerol, amino acids, and lactic acid (James et al. 2004).

Although aliphatic carboxylic acids are involved in the attraction of An. gambiae s.s. to humans, the role of the various acids remains elusive. Therefore, we aimed to examine the role of individual aliphatic carboxylic acids using synthetic blends. The carboxylic acids were tested individually (in combination with ammonia + lactic acid), and those that augmented the attractive effect were subsequently tested in combinations, using two different bioassay systems. Both optimization and subtraction experiments were performed in order to examine the contribution of individual carboxylic acids to a multicomponent blend.

\section{Methods and Materials}

Mosquitoes The colony of Anopheles gambiae sensu stricto (hereafter An. gambiae) was maintained at Wageningen University, The Netherlands, and originally obtained from Suakoko, Liberia (courtesy Prof. M. Coluzzi, Rome, Italy). The mosquitoes have been cultured in the laboratory since 1988 with blood meals from a human arm twice a week. The mosquito colony was kept in a climate room at $27 \pm 1^{\circ} \mathrm{C}, 80 \pm$ $5 \% \mathrm{RH}$ and a photo-scotophase of $12 / 12 \mathrm{hL} / \mathrm{D}$. Adult mosquitoes were maintained in $30 \times 30 \times 30 \mathrm{~cm}$ gauze cages with access to a $6 \%$ glucose solution on filter paper. Larvae were reared on tap water in plastic trays and fed daily with Tetramin ${ }^{\circledR}$ baby fish food (Melle, Germany). Pupae were collected daily and placed into adult cages for emergence.

Olfactometer A dual-port olfactometer, consisting of a flight chamber $(1.60 \times 0.66 \times 0.43 \mathrm{~m})$ with glass walls and a Luxan top, was used to study the behavioral responses of female mosquitoes to different odor stimuli. Pressurized air was charcoal filtered, humidified, and led through two Perspex mosquito trapping devices connected to two ports (4 cm diam and $28 \mathrm{~cm}$ apart) in the flight chamber (flow rate of $20.6 \pm 1.4 \mathrm{~cm} / \mathrm{s}$ ). The light from one tungsten light bulb (75 Watt) was filtered and scattered through a screen of yellow cloth hanging \pm $1 \mathrm{~m}$ above the flight chamber. This resulted in dim light of about 1 Lux in the olfactometer. The experimental room was maintained at $27 \pm 1{ }^{\circ} \mathrm{C}$ and a relative humidity of $61 \pm 9 \% \mathrm{RH}$. The temperature inside the flight chamber was $27 \pm 2.5^{\circ} \mathrm{C}$ and $64 \pm 9 \% \mathrm{RH}$. The air flowing out of the ports was maintained above $80 \% \mathrm{RH}$ and an air temperature of $27.4 \pm 1{ }^{\circ} \mathrm{C}$.

Odor Stimuli Tested In The Olfactometer To insure a continuous and constant odor concentration for each compound within the odor plumes from start until the end of each experiment, we used air sample bags for ammonia, glass bottles for L-(+)-lactic acid, and 15 aliphatic carboxylic acids (Geier et al. 1999; Bosch et al. 2000).

Ammonia was supplied in a similar way as described in Smallegange et al. (2005). One day before the experiments, $250 \mu \mathrm{l}$ of a $2.5 \%$ aqueous ammonia solution $(25 \%$ in water; analytical grade, Merck) were injected into an 801 Teflon air sample bag (SKC Gulf Coast Inc., Houston, TX, USA). Subsequently, the bag was filled with 601 of warm, humidified, and charcoal filtered pressurized air at least $17 \mathrm{~h}$ prior to experiments to allow evaporation of the solution. This procedure resulted in an ammonia concentration of $136 \mathrm{ppm}$ in the bag (Smallegange et al. 2005). During experiments, air pumps (Model 224-PCXR4, SKC Gulf Coast Inc., Houston, TX, USA) were used to lead air from the sample bag, through Teflon tubes $(7 \mathrm{~mm}$ in diam; Rubber B.V., Hilversum, The Netherlands), and into the trapping devices at a flow of $230 \mathrm{ml} / \mathrm{min}$. The flow of air was regulated by mechanical flow meters (Sho-Rate model GT1355; Brooks Instruments, Veenendaal, The Netherlands), and was mixed with the main air stream at a flow rate of approximately $23.5 \mathrm{1} / \mathrm{min}$.

L-(+)-lactic acid (90\% aqueous solution, analytical grade, Purac Bioquimica or $88-92 \%$ aqueous solution, Riedel-de Haën) (henceforth termed lactic acid) was mixed with the main air stream by tapping lactic acid vapor $(10 \mathrm{ml})$ from a $250 \mathrm{ml}$ glass bottle (Fisher Scientific B.V., 's Hertogenbosch, The Netherlands) with Teflon tubing. The flow rate was regulated to $15 \mathrm{ml} / \mathrm{min}$ by flow meters (Gilmont, Fisher Scientific B.V., 's Hertogenbosch, The Netherlands), which caused a lactic acid release comparable to that from a human hand (Smith et al. 1970; Geier et al. 1999). Since we applied lactic acid in a different way compared to previous experiments (Smallegange et al. 2005), we verified that it had no additional effect on the response of mosquitoes when added to ammonia.

Fifteen saturated aliphatic carboxylic acids $(\mathrm{C} 2-\mathrm{C} 16)$ of the highest purity grade available were used in the experiments: $>99 \%$ acetic acid (Sigma), 99\% propanoic acid 
(Sigma), 99\% 2-methylpropanoic acid (Sigma), $\geq 99 \%$ 3-methylbutanoic acid (Sigma), >99\% butanoic acid (Aldrich), $>99 \%$ pentanoic acid (Sigma), $\geq 99 \%$ hexanoic acid (Sigma), 98\% heptanoic acid (Sigma), $\geq 99 \%$ octanoic acid (Sigma), $\geq 97 \%$ nonanoic acid (Sigma), $>99 \%$ decanoic acid (Sigma), $\geq 99 \%$ dodecanoic acid (Sigma), $\geq 98 \%$ tridecanoic acid (Sigma), $>99 \%$ tetradecanoic acid (Sigma), $\geq 99 \%$ hexadecanoic acid (Sigma). Single, pure compounds [10 $\mathrm{ml}$ of a liquid compound (C2-C9) or $1 \mathrm{~g}$ of a solid compound $(\mathrm{C} 10-\mathrm{C} 16)]$ were added to a $250 \mathrm{ml}$ glass bottle (Fisher Scientific B.V., 's Hertogenbosch, The Netherlands). A charcoal-filtered, warm, humidified air stream was passed through the bottle and carried the vaporized compound at the desired flow rate through Teflon tubing and into the main stream through one of the trapping devices. Flow rates were regulated by Gilmont flow meters (Fisher Scientific B.V., 's Hertogenbosch, The Netherlands) at $0.5,5,50$, and $100 \mathrm{ml} / \mathrm{min}$. The calculated concentrations of the compounds in the air stream of the olfactometer are listed in Table S1 in the online supplement.

When tripartite blends were tested, the three compounds were mixed just before entering the trapping device. When more than one aliphatic carboxylic acid was part of the blend, a Perspex ring with 10 holes was attached upstream of each trapping device to be able to release each odor into the main air stream individually (Fig. 1). Tripartite blends were tested against ammonia alone. During experiments with multi-component blends, the multi-component odor blends were tested against the ammonia + lactic acid blend. Initially, we wanted to examine whether we could increase the attractiveness of ammonia-our best kairomone at that time. For this reason, we used ammonia alone as a control. In addition, we had observed that ammonia + lactic acid + a mixture of 12 carboxylic acids did not attract more mosquitoes than ammonia + lactic acid, but was more attractive than ammonia alone (Smallegange et al. 2005). Once we determined which individual carboxylic acids augmented the attractiveness of ammonia + lactic acid (Table 1), we continued our experiments with ammonia + lactic acid as the control, as we aimed for a better result than was found in Smallegange et al. (2005).

Olfactometer Tests Thirty female mosquitoes, 5-8 d-old, that had not received a blood meal, were randomly collected from their cage 14-18 $\mathrm{h}$ before the start of experiments. The mosquitoes were placed into a cylindrical release cage $(8-\mathrm{cm}$ diam, $10-\mathrm{cm}$ high) with access to tap water from damp cotton wool placed on top of the cage. Experiments were performed during the last $4 \mathrm{~h}$ of the dark period, when An. gambiae is normally active (Haddow and Ssenkubuge 1973; Maxwell et al. 1998; Killeen et al. 2006).

In each trial, test compounds were released into the air stream and a group of mosquitoes was set free from a release cage placed at the downwind end of the flight chamber of the olfactometer, $1.60 \mathrm{~m}$ from the two ports. Mosquitoes were left in the flight chamber for $15 \mathrm{~min}$. Female mosquitoes that had entered either of the trapping devices were counted at the end of the experiment, after anaesthetization with $100 \% \mathrm{CO}_{2}$. Mosquitoes remaining in the flight chamber were removed with a vacuum cleaner. After use, the trapping devices were washed with soapy water (CLY-MAX Heavy Duty Cleaner, Rogier Bosman Chemie B.V., Heijningen, The Netherlands), rinsed with tap water, and cleaned with cotton wool drenched in $70 \%$ ethanol (Merck).

The operator wore surgical gloves (Romed ${ }^{\circledR}$, powderfree vinyl) to avoid contamination of the equipment with human volatiles. Each trial started with new mosquitoes and clean trapping devices. An experiment testing a particular blend was repeated at least 6 times on different days. The sequence of test odors was randomized on the same day and between days. Test stimuli were alternated between

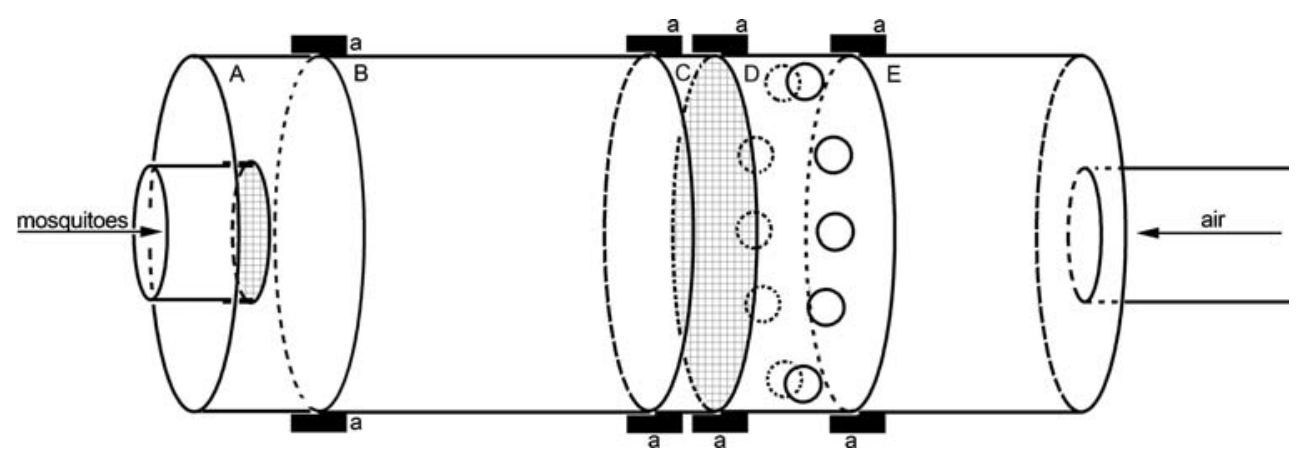

Fig. 1 An olfactometer trapping device is composed of three parts: $A$ : part with baffle where mosquitoes enter the device; $B$ : middle part device; $C$ : distal end sealed with metal gauze to prevent mosquito crossing. A Perspex ring $(D)$ with 10 holes for separate odor delivery. The end of the tube running from the glass bottle with an odor was inserted through one of the holes. Charcoal filtered, warm, humidified, pressurized air is led into the trapping device through $E$ (a: schematic representation of the couplings between the various parts, made to fit smoothly on each other to prevent air loss) 
Table 1 Effect of adding an individual carboxylic acid, at four flow rates $(\mathrm{ml} / \mathrm{min})$, to ammonia + lactic acid tested against ammonia alone in the dual-choice olfactometer. The result of the $\chi^{2}$-test ( $P$-value), trap entry response $(\%)$ and total number of mosquitoes released (n) are given for each twochoice test
A: significantly more mosquitoes in the trapping device baited with the tripartite blend compared to the trapping device baited with ammonia $\left(\chi^{2}\right.$-test, $\left.P<0.05\right)$. R: significantly fewer mosquitoes in the trapping device baited with the tripartite blend compared to the trapping device baited with ammonia ( $\chi^{2}$-test, $\left.P<0.05\right)$. Calculated concentrations of the compounds in the odor plume are given in the online supplement (Table S1).

n.t. not tested

\begin{tabular}{|c|c|c|c|c|}
\hline Carboxylic acid & $0.5 \mathrm{ml} / \mathrm{min}$ & $5 \mathrm{ml} / \mathrm{min}$ & $50 \mathrm{ml} / \mathrm{min}$ & $100 \mathrm{ml} / \mathrm{min}$ \\
\hline \multirow[t]{3}{*}{ Acetic acid (C2) } & $P=0.25$ & $P=0.24$ & $P=0.85$ & $P=0.56$ \\
\hline & $24.1 \%$ & $22.5 \%$ & $14.4 \%$ & $15.3 \%$ \\
\hline & $n=199$ & $n=262$ & $n=201$ & $n=177$ \\
\hline \multirow[t]{3}{*}{ Propanoic acid (C3) } & $P=0.13$ & $P<0.001 \mathrm{~A}$ & $P=0.59$ & $P=0.001 \mathrm{~A}$ \\
\hline & $15.7 \%$ & $21.2 \%$ & $8.8 \%$ & $20.8 \%$ \\
\hline & $n=178$ & $n=156$ & $n=160$ & $n=154$ \\
\hline \multirow[t]{3}{*}{ 2-Methylpropionic acid (2mC3) } & $P=1.00$ & $P=0.38$ & $P=0.34$ & $P=0.22$ \\
\hline & $8.2 \%$ & $18.9 \%$ & $30.5 \%$ & $39.9 \%$ \\
\hline & $n=170$ & $n=175$ & $n=174$ & $n=198$ \\
\hline \multirow[t]{3}{*}{ Butanoic acid (C4) } & $P=0.03 \mathrm{~A}$ & $P=0.69$ & $P=0.53$ & $P=0.47$ \\
\hline & $24.7 \%$ & $11.6 \%$ & $6.1 \%$ & $11.0 \%$ \\
\hline & $n=150$ & $n=225$ & $n=164$ & $n=155$ \\
\hline \multirow[t]{3}{*}{ 3-Methylbutanoic acid (3mC4) } & $P=0.008 \mathrm{~A}$ & $P=0.64$ & n.t. & n.t. \\
\hline & $16.6 \%$ & $10.2 \%$ & & \\
\hline & $n=169$ & $n=176$ & & \\
\hline \multirow[t]{3}{*}{ Pentanoic acid (C5) } & $P=0.13$ & $P=0.32$ & $P=0.86$ & $P=0.01 \mathrm{~A}$ \\
\hline & $6.3 \%$ & $20.6 \%$ & $18.5 \%$ & $39.4 \%$ \\
\hline & $n=174$ & $n=175$ & $n=178$ & $n=175$ \\
\hline \multirow[t]{3}{*}{ Hexanoic acid (C6) } & $P=0.003 \mathrm{R}$ & $P=0.66$ & $P=0.88$ & $P=0.73$ \\
\hline & $19.7 \%$ & $17.5 \%$ & $26.3 \%$ & $15.7 \%$ \\
\hline & $n=234$ & $n=468$ & $n=179$ & $n=464$ \\
\hline \multirow[t]{3}{*}{ Heptanoic acid (C7) } & $P=0.03 \mathrm{~A}$ & $P=0.005 \mathrm{R}$ & $P=0.007 \mathrm{R}$ & $P=0.85$ \\
\hline & $8.8 \%$ & $11.0 \%$ & $13.5 \%$ & $15.4 \%$ \\
\hline & $n=226$ & $n=164$ & $n=170$ & $n=175$ \\
\hline \multirow[t]{3}{*}{ Octanoic acid (C8) } & $P=0.30$ & $P=0.47$ & $P<0.001 \mathrm{~A}$ & $P=0.23$ \\
\hline & $13.5 \%$ & $16.0 \%$ & $29.5 \%$ & $29.2 \%$ \\
\hline & $n=170$ & $n=187$ & $n=193$ & $n=195$ \\
\hline \multirow[t]{3}{*}{ Nonanoic acid (C9) } & $P=0.78$ & $P=0.41$ & $P=0.16$ & $P=0.53$ \\
\hline & $7.6 \%$ & $13.5 \%$ & $4.5 \%$ & $5.6 \%$ \\
\hline & $n=170$ & $n=178$ & $n=177$ & $n=177$ \\
\hline \multirow[t]{3}{*}{ Decanoic acid (C10) } & $P=0.32$ & $P=1.00$ & $P=1.00$ & $P=0.53$ \\
\hline & $4.1 \%$ & $6.9 \%$ & $3.7 \%$ & $4.5 \%$ \\
\hline & $n=219$ & $n=232$ & $n=215$ & $n=221$ \\
\hline \multirow[t]{3}{*}{ Dodecanoic acid (C12) } & $P=0.47$ & $P=1.00$ & $P=1.00$ & $P=0.78$ \\
\hline & $9.4 \%$ & $8.9 \%$ & $4.4 \%$ & $7.3 \%$ \\
\hline & $n=180$ & $n=180$ & $n=180$ & $n=178$ \\
\hline \multirow[t]{3}{*}{ Tridecanoic acid (C13) } & $P=0.26$ & $P=0.82$ & $P=0.09$ & $P=0.58$ \\
\hline & $23.4 \%$ & $11.3 \%$ & $25.4 \%$ & $17.3 \%$ \\
\hline & $n=167$ & $n=168$ & $n=169$ & $n=168$ \\
\hline \multirow[t]{3}{*}{ Tetradecanoic acid (C14) } & $P=0.02 \mathrm{~A}$ & $P=0.04 \mathrm{~A}$ & $P=0.007 \mathrm{~A}$ & $P=0.01 \mathrm{~A}$ \\
\hline & $8.7 \%$ & $11.2 \%$ & $11.5 \%$ & $12.7 \%$ \\
\hline & $n=173$ & $n=170$ & $n=174$ & $n=173$ \\
\hline \multirow[t]{3}{*}{ Hexadecanoic acid (C16) } & $P=0.06$ & $P=0.37$ & $P=0.74$ & $P=0.80$ \\
\hline & $5.7 \%$ & $6.3 \%$ & $5.2 \%$ & $8.5 \%$ \\
\hline & $n=175$ & $n=176$ & $n=172$ & $n=177$ \\
\hline
\end{tabular}


right and left ports in different replicates to rule out any positional effects. Experiments in which only clean air was fed into the olfactometer through both ports were done to test the symmetry of the trapping system.

Trapping Experiments in Screen Cage To assess the performance of some of the blends as a lure, we conducted laboratory experiments with volatile baited MM-X traps in a textile screen cage $(233 \times 250 \times 330 \mathrm{~cm}$; Howitec Netting BV, Bolsward, The Netherlands) inside a climate-controlled room $\left(22.7 \pm 1.1^{\circ} \mathrm{C}\right.$ and $\left.52.4 \pm 7.4 \% \mathrm{RH}\right)$. Two Mosquito Magnet-X (MM-X) traps (American Biophysics Corp., U.S.A.) (Kline 1999) were placed inside the cage at $2 \mathrm{~m}$ distance from each other.

Initially, a 9-compound blend (blend A) was tested. To compose this blend, $500 \mu \mathrm{l}$ of a liquid pure compound or $500 \mathrm{mg}$ of a solid compound (tetradecanoic acid) were put into individual low density polyethylene sachets (LDPE; $6 \times 6 \mathrm{~cm}$; Audion Elektro, The Netherlands; Torr et al. 1997) with a thickness of $0.1 \mathrm{~mm}$ (ammonia, lactic acid, heptanoic, octanoic, and tetradecanoic acid) or in a closed LDPE tube $(32 \times 14 \mathrm{~mm}$, Kartell, $3.5 \mathrm{ml}$; Fisher Emergo, The Netherlands) within an LDPE sachet with a thickness of $0.2 \mathrm{~mm}$ (this delivery method was used for the 4 most volatile aliphatic carboxylic acids (i.e., propanoic, butanoic, 3-methylbutanoic, and pentanoic acids) to reduce their release to a higher extent than would have been possible with a sachet only). Only ammonia was diluted with distilled water (to $2.5 \%$ ). The sachets were applied inside the central, black tube of the MM-X traps using odorless tape (3M $\mathrm{M}^{\mathrm{TM}}$ Double Coated Tape 400). Air flow was created by a fan on top of this tube taking the headspace of the test blend downwards and outside the MM-X trap. Second, a tripartite blend was tested (blend B). Since we calculated that the release rates of the aliphatic carboxylic acids in blend $\mathrm{A}$ were at least two times higher than calculated for the multi-component blends tested in the optimization and subtraction olfactometer experiments, the 3 LDPE sachets (each with a thickness of $0.1 \mathrm{~mm}$ ) containing the separate components of blend $\mathrm{B}$ were made as small as possible $(2.5 \times 2.5 \mathrm{~cm})$ to reduce evaporation (Torr et al. 1997). One hundred $\mu 1$ of a liquid pure compound (ammonia, lactic acid) or $50 \mathrm{mg}$ of a solid compound (tetradecanoic acid) were put into individual LDPE sachets. The amount of a compound within a sachet does not affect the evaporation rate of the compound through the LDPE material, whereas the surface of a LDPE sachet does (Torr et al. 1997). When blend B was applied, ammonia was not diluted (25.0\%). Both blend A and $\mathrm{B}$ were tested against a blend of ammonia + lactic acid. Evaporation rates of the compounds were measured by weighing the LDPE sachets before and after experiments (see Table $\mathrm{S} 1$ in the online supplement).
Fifty female mosquitoes, 5-8 d-old, which had not received a blood meal, were randomly collected $14-18 \mathrm{~h}$ before the start of experiments. They were placed into a cylindrical release cage (diam $8 \mathrm{~cm}$, height $17.5 \mathrm{~cm}$ ) with access to tap water from damp cotton wool placed on top of the cage. The mosquitoes were set free from the release cage in the center of the screen cage. After $4 \mathrm{~h}$, the MM-X traps were closed and transferred into a freezer to kill the mosquitoes. These experiments were performed during the last $4 \mathrm{~h}$ of the dark period. Each two-choice test was repeated either 4 or 6 times, alternating the position of each treatment every experimental day. Surgical gloves were worn to avoid contamination of equipment with human volatiles. Experiments with unbaited traps in the MM-X setup were done 6 times to test the symmetry of the trapping system.

Statistical Analysis For each two-choice assay, a Chisquare test was used to analyze whether the total number (i.e., sum of all replicates; a comparison of data collected on different days revealed no heterogeneity) of mosquitoes trapped in the treatment trapping device (of either olfactometer or MM-X trap) and the total number that was trapped in the control trapping device (of olfactometer or MM-X trap) differed from a 1:1 distribution. Effects were considered to be significant at $P<0.05$. The number of female mosquitoes caught in both trapping devices divided by the number of mosquitoes that flew out of the release cage is expressed as the trap entry response (TER).

\section{Results}

Initial Experiments The combined results of all dual-choice olfactometer experiments with clean air from both ports together show that equal numbers of mosquitoes were caught in both trapping devices (Chi-square test, $d f=1, P=$ 0.16 ). This is proof that the olfactometer was symmetrical and that no positional bias interfered. Even when no volatile source was present, small numbers of mosquitoes flew into the trapping device. However, the TER was low $(5.9 \%, N=853)$.

The combination of ammonia (136 ppm) + lactic acid $(15 \mathrm{ml} / \mathrm{min}$ ) caught mosquitoes in numbers similar to those caught by ammonia alone $(P=0.14, N=324$, TER $=28.4 \%)$. Based on these results and the synergism between ammonia, lactic acid, and a blend of carboxylic acids that we reported previously (Smallegange et al. 2005), we decided to test single carboxylic acids by adding them to the blend of ammonia + lactic acid and examine the effect of this synthetic tripartite blend on the response of An. gambiae compared to the effect of ammonia alone. 
Individual Aliphatic Carboxylic Acids First, 15 aliphatic carboxylic acids were tested in the dual-choice olfactometer at four different release rates, for their ability to increase the attractiveness of the blend of ammonia + lactic acid compared to ammonia alone. Seven of the carboxylic acids had no effect at any of the four concentrations (Table 1), namely acetic, 2-methylpropanoic, nonanoic, decanoic, dodecanoic, tridecanoic, and hexadecanoic acids ( $\mathrm{C} 2$, 2mC3, C9, C10, C12, C13, and C16). Depending on the release rate applied, hexanoic acid had either no effect or significantly decreased the number of mosquitoes flying into trapping devices baited with the tripartite blend compared to those flying into the trapping devices baited with ammonia only $(P=0.003$ at the lowest concentration tested).

Adding propanoic, butanoic, 3-methylbutanoic, pentanoic, heptanoic, octanoic, or tetradecanoic acids $(\mathrm{C} 3, \mathrm{C} 4$, $3 \mathrm{mC} 4, \mathrm{C} 5, \mathrm{C} 7, \mathrm{C} 8$, and $\mathrm{C} 14)$ to the ammonia + lactic acid blend resulted in an increase in the number of mosquitoes flying into the trapping device at one or more of the tested concentrations. The tripartite blend was more attractive than ammonia when butanoic, 3methylbutanoic, pentanoic, heptanoic, or octanoic acids were part of the blend at either the lowest (flow rate $0.5 \mathrm{ml} / \mathrm{min}, \mathrm{C} 4,3 \mathrm{MC} 4$ and $\mathrm{C} 7, P<0.05$ ), the second highest $(50 \mathrm{ml} / \mathrm{min}, \mathrm{C} 8, P<0.001)$ or highest concentration $(100 \mathrm{ml} / \mathrm{min}, \mathrm{C} 5, P=0.01)$. The second lowest and the highest concentrations of propanoic acid ( 5 and $100 \mathrm{ml} / \mathrm{min}$ ) enhanced the attraction of ammonia + lactic acid $(P \leq$ 0.001). All four concentrations of tetradecanoic acid increased the attraction to ammonia + lactic acid compared with the attraction to ammonia alone $(P<0.05)$. At the lowest concentration $(0.5 \mathrm{ml} / \mathrm{min})$, heptanoic acid increased the attractiveness of ammonia in combination with lactic acid $(P=0.03)$. However, fewer mosquitoes were caught at the two intermediate heptanoic acid concentrations (5 and $50 \mathrm{ml} / \mathrm{min})$ added to ammonia + lactic acid $(P=0.005$ and 0.007 , respectively).

Optimization Of A Multi-Component Blend Based on previous odor blend experiments, a blend of ammonia + lactic acid and the 7 aliphatic carboxylic acids $(\mathrm{C} 3, \mathrm{C} 4$, $3 \mathrm{mC} 4, \mathrm{C} 5, \mathrm{C} 7, \mathrm{C} 8$, and C14) was prepared. This blend enhanced the attractiveness of ammonia when combined in a tripartite blend with lactic acid. Dual-choice olfactometer experiments were performed in which the concentrations of the 7 aliphatic carboxylic acids within this blend were varied in order to examine their effectiveness as mosquito attractants. In total, odor blends were tested in 6 different compositions in which the release rates varied between blends (see Table 2). Two of these experimental blends (blend 1 and blend 4) elicited significantly stronger attractions to the blend of 9 compounds (i.e., the 'complex blend') than to ammonia + lactic acid only (the 'control blend') (Table 2, $P<0.001$ ). The other blends were equally attractive as the control blend (Table 2, $P>0.05$ ).

Subtraction Experiments Blend 4, containing all 7 of the aliphatic carboxylic acids [each applied at the lowest dose $(0.5 \mathrm{ml} / \mathrm{min})$ ], was selected for subtraction experiments. The blend minus one of the 7 acids was tested against the control blend (ammonia + lactic acid) to determine the contribution of each individual carboxylic acid on the attractiveness of the complex blend.

Removal of 3-methylbutanoic acid from the complex blend increased the blend attractiveness significantly (Table 3, $P=0.01$ ). When tetradecanoic acid was removed, the test blend attracted fewer mosquitoes into the trapping device than the control blend $(P=0.02)$. Elimination of
Table 2 Optimization experiments with carboxylic acids in the dualchoice olfactometer. The left part of the table shows the flow rates for each individual aliphatic carboxylic acid within an odor blend consisting of nine components (ammonia + lactic acid + seven carboxylic acids). The total numbers of mosquitoes caught in the trapping device baited with the test blend (T) and in the trapping device baited with the control blend (C) are given. Also the result of the $\chi^{2}$-test, total number of mosquitoes released $(\mathrm{N})$ and trap entry response (TER) are given for each blend

\begin{tabular}{|c|c|c|c|c|c|c|c|c|c|c|c|c|}
\hline \multirow[t]{2}{*}{ Blend } & \multicolumn{7}{|c|}{ Flow rate $(\mathrm{ml} / \mathrm{min})$} & \multirow[t]{2}{*}{$\mathrm{T}$} & \multirow[t]{2}{*}{$\mathrm{C}$} & \multirow[t]{2}{*}{$\chi^{2}$-test } & \multirow[t]{2}{*}{$\mathrm{N}$} & \multirow[t]{2}{*}{ TER } \\
\hline & $\mathrm{C} 3$ & $3 \mathrm{mC} 4$ & $\mathrm{C} 4$ & $\mathrm{C} 5$ & $\mathrm{C} 7$ & $\mathrm{C} 8$ & $\mathrm{C} 14$ & & & & & \\
\hline 1 & 100 & 0.5 & 0.5 & 100 & 0.5 & 50 & 50 & 56 & 25 & $P<0.001 \mathrm{~A}$ & 715 & $11.3 \%$ \\
\hline 2 & 50 & 0.5 & 100 & 0.5 & 0.5 & 100 & 100 & 3 & 2 & $P=0.65$ & 169 & $3.0 \%$ \\
\hline 3 & 0.5 & 0.5 & 0.5 & 0.5 & 0.5 & 5 & 100 & 9 & 10 & $P=0.82$ & 174 & $10.9 \%$ \\
\hline 4 & 0.5 & 0.5 & 0.5 & 0.5 & 0.5 & 0.5 & 0.5 & 30 & 9 & $P<0.001 \mathrm{~A}$ & 548 & $7.1 \%$ \\
\hline 5 & 0.06 & 0.06 & 0.06 & 0.06 & 0.06 & 0.06 & 0.06 & 9 & 16 & $P=0.80$ & 171 & $8.8 \%$ \\
\hline 6 & 5 & 0.5 & 0.5 & 5 & 0.5 & 5 & 5 & 13 & 5 & $P=0.06$ & 146 & $12.3 \%$ \\
\hline
\end{tabular}

A: significantly more mosquitoes in the trapping device baited with the complex blend compared to the trapping device baited with ammonia + lactic acid $\left(\chi^{2}\right.$-test, $\left.P<0.05\right)$. 
Table 3 Subtraction experiments performed in the dual-choice olfactometer. The total numbers of mosquitoes caught in the trapping device baited with the test blend (T) and in the trapping device baited with the control blend (C) are given. Also the result of the $\chi^{2}$-test, total number of mosquitoes released $(\mathrm{N})$ and trap entry response (TER) are given for each blend

\begin{tabular}{lrrllr}
\hline Blend & $\mathrm{T}$ & $\mathrm{C}$ & $\chi^{2}$-test & $\mathrm{N}$ & TER \\
\hline Blend 4 & 15 & 15 & $P=1.000$ & 338 & $8.9 \%$ \\
Blend 4 -C3 & 2 & 7 & $P=0.10$ & 171 & $5.3 \%$ \\
Blend 4 -C4 & 7 & 10 & $P=0.47$ & 164 & $10.4 \%$ \\
Blend 4 -3mC4 & 13 & 3 & $P=0.01 \mathrm{~A}$ & 165 & $9.7 \%$ \\
Blend 4 -C5 & 1 & 6 & $P=0.06$ & 171 & $4.1 \%$ \\
Blend 4 -C7 & 12 & 12 & $P=1.000$ & 171 & $14.0 \%$ \\
Blend 4 -C8 & 9 & 6 & $P=0.44$ & 160 & $9.4 \%$ \\
Blend 4 -C14 & 4 & 14 & $P=0.02 \mathrm{R}$ & 165 & $10.9 \%$ \\
\hline
\end{tabular}

A: significantly more mosquitoes in the trapping device baited with the complex blend compared to the trapping device baited with ammonia + lactic acid $\left(\chi^{2}\right.$-test, $\left.P<0.05\right)$.

$\mathrm{R}$ : significantly fewer mosquitoes in trapping device baited with complex blend compared to trapping device baited with ammonia + lactic acid $\left(\chi^{2}\right.$-test, $\left.P<0.05\right)$

pentanoic acid may have reduced the attractiveness of the complex blend $(P=0.06)$, but this effect was only marginally significant. Removal of the other four aliphatic carboxylic acids had no significant effect.

Effect of 3-Methylbutanoic Acid Based on the previous results, the effect of 3-methylbutanoic acid was examined by using a blend of ammonia + lactic acid + tetradecanoic acid (shown to have an important role within the complex blend, Table 3$)$ and also propanoic and pentanoic acid ( $P$-value in subtraction experiments $\leq 0.10$, Table 3 ). In this series of experiments, blend 4 again was significantly more attractive than the control blend (ammonia+lactic acid) (Table 4, $P=$ $0.02)$. A blend of ammonia + lactic + propanoic + pentanoic + tetradecanoic acids was as attractive as the control $(P=0.56)$. Adding 3-methylbutanoic acid to the latter blend at a flow rate of $0.5 \mathrm{ml} / \mathrm{min}$ did not alter the attractiveness, whereas reducing the flow rate to $0.05 \mathrm{ml} / \mathrm{min}$ increased attractiveness close to a marginally significant level $(P=0.06)$.

Trapping Experiments Dual-choice experiments were conducted with MM-X traps baited with either a blend of ammonia + lactic acid + tetradecanoic acid or a blend of ammonia + lactic acid + the 7 carboxylic acids. These two blends were tested against the control blend containing ammonia + lactic acid. Traps baited with the tripartite blend and the 9-component blend trapped significantly more mosquitoes than traps baited with the control blend (Table 5, $P<0.001)$. The two traps together caught $44.0 \%$ and $53.3 \%$ of the released mosquitoes ( $N=298$ and 199, respectively). Two unbaited MM-X traps together caught $35.2 \%$ of the mosquitoes that left the release cage $(N=585)$. These experiments without odor baits demonstrated that the system was symmetrical $(P=0.40)$.

\section{Discussion}

Our results show that aliphatic carboxylic acids with defined chain lengths (C3-C8 and C14) enhance the attractiveness of ammonia to An. gambiae mosquitoes in a dose-dependent manner when applied in a tripartite blend with lactic acid. These compounds are likely to serve as kairomones in the host-seeking behavior of An. gambiae. As several of these compounds also affect the host-seeking behavior of other mosquito species-e.g., Ae. aegypti (Bosch et al. 2000) and Culex quinquefasciatus Say, another mosquito species that acts as a vector of human disease (Allan et al. 2006; Puri et al. 2006), our findings lend further credence to the kairomonal role of aliphatic carboxylic acids present on the human skin for anthropophilic mosquitoes.

Individual Aliphatic Carboxylic Acids The carboxylic acids that enhanced the attractiveness of the blend of ammonia and lactic acid to An. gambiae all occur in human skin
Table 4 Effect of adding four carboxylic acids in different combinations to ammonia + lactic acid tested against ammonia + lactic acid in the dual-choice olfactometer. The total numbers of mosquitoes caught in the trapping device baited with the test blend $(\mathrm{T})$ and in the trapping device baited with the control blend $(\mathrm{C})$ are given. Also the result of the $\chi^{2}$-test, total number of mosquitoes released $(\mathrm{N})$ and trap entry response (TER) are given for each blend

\begin{tabular}{|c|c|c|c|c|c|}
\hline Blend & $\mathrm{T}$ & $\mathrm{C}$ & $x^{2}$-test & $\mathrm{N}$ & TER \\
\hline Blend 4 & 29 & 14 & $P=0.02 \mathrm{~A}$ & 258 & $16.7 \%$ \\
\hline ammonia + lactic acid $+\mathrm{C} 3+\mathrm{C} 5+\mathrm{C} 14$ & 15 & 12 & $P=0.56$ & 346 & $7.8 \%$ \\
\hline ammonia + lactic acid $+\mathrm{C} 3+3 \mathrm{mC}_{(0.5)}+\mathrm{C} 5+\mathrm{C} 14$ & 11 & 12 & $P=0.83$ & 84 & $27.4 \%$ \\
\hline ammonia + lactic acid $+\mathrm{C} 3+3 \mathrm{mC}_{(0.05)}+\mathrm{C} 5+\mathrm{C} 14$ & 22 & 11 & $P=0.06$ & 261 & $12.6 \%$ \\
\hline
\end{tabular}

$3 \mathrm{mC4}_{(0.5)}$ and $3 \mathrm{mC4}_{(0.05)}$ : 3-methylbutanoic acid applied at an air flow of 0.5 or $0.05 \mathrm{ml} / \mathrm{min}$ passing through the glass bottle containing this compound. All other aliphatic carboxylic acids were applied at a flow rate of $0.5 \mathrm{ml} / \mathrm{min}$. A: significantly more mosquitoes in the trapping device baited with the complex blend compared to the trapping device baited with ammonia + lactic acid $\left(\chi^{2}\right.$-test, $\left.P<0.05\right)$. 
Table 5 Blends tested in dual-choice screen cage experiments with MM-X traps. A MM-X trap was baited with one of the 2 test blends (T); the other MM-X trap was baited with ammonia and lactic acid (C). The total numbers of mosquitoes caught in the trap baited with the

\begin{tabular}{llllr}
\hline Blend & T & C & $\chi^{2}$-test & N \\
\hline A. ammonia + lactic acid + 7 carboxylic acids & 85 & 21 & $P<0.001$ A & 199 \\
B. ammonia + lactic acid + tetradecanoic acid & 87 & 44 & $P<0.001$ A & 298 \\
\hline
\end{tabular}

A: significantly more mosquitoes in the trapping device baited with the test blend compared to the trapping device baited with the control blend $\left(\chi^{2}\right.$-test, $\left.P<0.05\right)$. The release rate of each compound applied in LDPE sachets are given in the online supplement (Table S1).

emanations (Bernier et al. 2000; Healy and Copland 2000; Curran et al. 2005; Penn et al. 2007; Gallagher et al. 2008). Our results suggest that only few of these carboxylic acids, depending on their chain length and concentration, contribute to the attractiveness of An. gambiae to humans. Both attractive and repellent compounds might be involved in the differential host preference of this mosquito species (Qiu et al. 2004, 2006a). We have documented here that aliphatic carboxylic acids can act in both ways depending on concentration and molecular structure.

Knols et al. (1997) reported that 12 of the 15 acids tested also in this study comprised an attractive synthetic blend. However, the same blend was repellent to An. gambiae in a later study, but when combined with ammonia + lactic acid, produced an attractive blend in a synergistic fashion (Smallegange et al. 2005). Acetic acid levels in fresh sweat were higher than in incubated human sweat, the latter being more attractive to An. gambiae (Meijerink et al. 2000). Propanoic and hexanoic acids were detected in fresh sweat, but not incubated sweat. Levels of butanoic acid were similar in fresh and incubated sweat. 3-Methylbutanoic acid does occur in incubated human sweat, but in small amounts. We found that propanoic, butanoic, and 3methylbutanoic acid affect the host-seeking response of An. gambiae.

At the lowest dose, heptanoic acid increased the attractiveness of ammonia + lactic acid, whereas fewer mosquitoes were attracted to the two intermediate doses (Table 1). This confirms that not only the molecular structure of the odor, but also its dosage determines whether an odor acts as an attractant or a repellent (Vale and Hall 1985). Heptanoic, nonanoic, and tridecanoic acids, the latter two inactive in this study, were not present in the 12 carboxylic acid-containing synthetic mixture used as attractant by Knols et al. (1997) and which had a synergistic effect in combination with ammonia and lactic acid (Smallegange et al. 2005).

A tripartite blend of ammonia + lactic + and hexanoic acid was found to have a synergistic effect in Y-tube olfactometer tests with An. gambiae (Smallegange et al. 2002). Olfactory neurons tuned to aliphatic carboxylic acids test blend $(\mathrm{T})$ and in the trap baited with the control blend $(\mathrm{C})$ are given. Also the result of the $\chi^{2}$-test, total number of mosquitoes released $(\mathrm{N})$ and trap entry response (TER) are given for each blend were found to innervate antennal sensilla trichodea in $A n$. gambiae (Meijerink et al. 2000; Qiu et al. 2006b). Antennal grooved peg sensilla contain neurons sensitive to pentanoic and hexanoic acids. Hexanoic acid also elicited electrophysiological responses from olfactory neurons in trichoid sensilla (Qiu et al. 2006b). Carboxylic acids eliciting an electrophysiological response, but not showing a behavioral effect in this study, may be involved in other behaviors of this mosquito species.

$\mathrm{C} 1-3, \mathrm{C} 5-8$, and $\mathrm{C} 13-18$ carboxylic acids enhance the attractiveness of lactic acid to Ae. aegypti (Bosch et al. 2000). Several carboxylic acids (C2-18) attract high numbers of female $C x$. quinquefasciatus in Y-tube olfactometer experiments or induce landing responses when tested individually (Allan et al. 2006; Puri et al. 2006). Bosch et al. (2000) found that hexanoic acid augmented the attractiveness of lactic acid to Ae. aegypti. A blend of ammonia, lactic acid, and hexanoic acid is now commercially available (BioGents, Germany; Kröckel et al. 2006) and shown to have potential as an attractant for Ae. aegypti (Kröckel et al. 2006; Williams et al. 2006a, b, c). However, in the present study with An. gambiae, none of the hexanoic acid doses enhanced attractiveness. In fact, repellency occurred at the lowest dose (Table 1). Attempts to reduce the hexanoic acid concentration from a glass bottle $(0.05 \mathrm{ml} / \mathrm{min}$ and lower) did not have any effect on the attractiveness of the combination of ammonia, lactic acid, and hexanoic acid compared to ammonia alone. Also, flow rates of $25 \mathrm{ml} / \mathrm{min}$ and $230 \mathrm{ml} / \mathrm{min}$ had no effect (data not shown). When 8 doses of hexanoic acid were applied to a sand-blasted glass slide (see Smallegange et al. 2005), no attraction was found (in combination with ammonia and lactic acid against ammonia and solvents (diethyl ether; Merck, $\geq 99.8 \%$ ) to dilute hexanoic acid (Sigma, $\geq 99 \%$ ), and ethanol (Merck, $\geq 99.8 \%$ ) to dilute L(+)-lactic acid (sodium salt, Sigma, 98\%) (data not shown). Therefore, we conclude that hexanoic acid is not important to An. gambiae in attracting host-seeking females from a distance.

Several carboxylic acids showed neither an attractive nor a repellent effect toward An. gambiae females at any of the concentrations tested. We do not know the actual concentra- 
tion of the acids encountered by mosquitoes in our bioassays or when engaged in host-seeking at varying distances from the host under typical host-seeking conditions. It is possible that the acids were tested at concentrations (see Table S1 in the online supplement) outside of the range that naturally occur in the volatile blends released by humans. Further study of the absolute concentration of humanproduced volatiles in the odor plume is required to clarify the role of single carboxylic acids as kairomones for blood feeding insects.

Optimization Experiments Both the optimization and the trapping experiments with the odor blends consisting of nine compounds showed that the blend containing ammonia, lactic acid, and the 7 carboxylic acids enhanced the effect of ammonia in the first experiment, and is attractive to An. gambiae. However, the concentration and/or the ratio of carboxylic acids, are crucial factors as can be concluded from the optimization experiments (Table 2). Apparently, the flow rates of the carboxylic acids in blend 5 were too low to induce an effect. When comparing blend 1, 2, and 4, it is likely that the flow rates of butanoic, octanoic, and/or tetradecanoic acid in blend 2 were too high. It is surprising that blend 3 showed no effect. This suggests that the flow rate of tetradecanoic acid was too high, although this seems to contradict the results obtained with this carboxylic acid in the first experiment. It may be that the highest concentration at which tetradecanoic acid is attractive depends on the presence of other carboxylic acids.

\section{Subtraction Experiments And The Effect Of 3-Methylbutanoic} Acid Blend 4 was no longer more attractive than the control blend when we initiated the series of subtraction experiments (Table 3), unlike the results presented in Table 2. However, the subtraction experiments are valid, since the effect of eliminating an individual aliphatic carboxylic acid on the attractiveness of the complex blend is clear. In addition, blend 4 was attractive in a series of olfactometer experiments conducted after the subtraction studies (Table 4). Moreover, MM-X traps baited with this blend attracted 4 times more mosquitoes than the control blend in a larger set-up (Table 5). Therefore, we conclude that blend 4 is attractive to $A n$. gambiae and that the blend components jointly affect hostseeking behavior.

Individual removal of propanoic, butanoic, pentanoic, heptanoic, or octanoic acid had no effect on the attractiveness of the complex blend suggesting that these 5 carboxylic acids do not play a significant role in the attractiveness of the complex blend and might be exchanged by one another.

Elimination of tetradecanoic acid made the complex blend less attractive than the control blend, whereas the complex blend without 3-methylbutanoic acid was significantly more attractive than the control blend. This suggests that 3-methylbutanoic acid has a repellent or inhibitory effect. On the other hand, a low dose of 3-methylbutanoic acid $(0.05 \mathrm{ml} / \mathrm{min})$ also appeared to have a positive effect (although not quite significant) compared with the blend of 5 compounds (Table 4). The effect of 3-methylbutanoic acid seems to depend on the dose of this compound and the other carboxylic acids in the blend (see also Table 1). However, strictly adhering to the significance level of $5 \%$, none of the blends with either 3 or 4 aliphatic carboxylic acids mentioned in Table 4 had the same effect as blend 4 $(P=0.02)$. In other words, these four aliphatic carboxylic acids cannot replace the 7 aliphatic carboxylic acids in blend 4 to enhance the attractiveness of ammonia + lactic acid. Apparently (taking all results together), interactions between aliphatic carboxylic acids in odor blends affect the "active" composition of the blend, which we cannot fully elucidate based on our results. Combining the subtraction results with the result of the first experiment where tetradecanoic acid increased the attractiveness of ammonia when lactic acid was present at all flow rates, suggests that it is an important component in behaviorally significant odor blends for An. gambiae. This aliphatic carboxylic acid was also found to be more abundant (almost twice as much) in the odor profiles of two people that were more attractive to An. gambiae than two people with lower amounts of tetradecanoic acid. In addition, tetradecanoic acid was a major constituent in the odor profiles of these four people as determined by sampling skin residues on small glass beads (Qiu et al. 2006a). The residues were desorbed by placing the beads into a thermal desorption unit combined with a cryo-focussing system and then analyzed by GC-MS (A.M. Galimard, personal communication).

Trapping Experiments The trapping experiments showed that a complex blend of 9 compounds is more attractive than the control blend (ammonia + lactic acid), which is in agreement with the results of the olfactometer assays. A catch rate of about $42.7 \%$ of the mosquitoes released by the MM-X trap baited with the complex blend compared to $17.6 \%$ per trap in experiments with two unbaited traps is a promising finding. Semi-field and field experiments should be conducted to reveal the potential of this blend as a bait for wild mosquitoes in Africa. The MM-X trap baited with the tripartite blend also caught more mosquitoes than the MM-X trap baited with the bipartite blend, suggesting that despite its low volatility, tetradecanoic acid increases the attractiveness of ammonia and lactic acid in two different assays.

Comparisons of the release rates that were calculated based on physicochemical parameters and the gravimetrically determined evaporation rates (Table $\mathrm{S} 1$ in the online supplement) of compounds applied in the olfactometer and in the MM-X traps, respectively, seem to show differences 
in the concentrations applied in the two bioassays. However, it is hard to estimate the exact concentrations encountered by mosquitoes, considering the differences between the two bioassays (e.g., sizes of the experimental arenas in which experiments have been conducted and the release methods used for the test compounds).

The tripartite blend caught about $29.2 \%$ of the total number of mosquitoes released into the experimental room. When the attractiveness of the 9-component blend is compared statistically with the tripartite blend, a significant difference is not found $[P=0.73$; GLM (binomial, link in logit); calculated for the ratio of (treatment)/(treatment + control); mean $0.60 \pm 0.39$ (9-component blend) and $0.66 \pm$ 0.11 (tripartite blend)]. Thus, the trapping experiments show, in addition to olfactometer data, that tetradecanoic acid in combination with ammonia and lactic acid may be used as a reliable basic blend to which other candidate kairomones for An. gambiae can be added. Although semifield and field experiments have to be conducted to see how well this tripartite blend performs under African conditions, it seems likely that the blend efficacy can be improved by adjusting the concentrations of each compound and by addition of compounds other than aliphatic carboxylic acids (R.C. Smallegange, unpublished data).

In summation, our results demonstrate that several aliphatic carboxylic acids elicit a significant odormediated behavioral preference in the malaria mosquito An. gambiae. They also enhance the attractive effectiveness of the ammonia and lactic acid mixture. Tetradecanoic acid emerges as a crucial compound and is attractive over a broad concentration range. In contrast, 3-methylbutanoic acid reduced the trap entry response when present in a blend of ammonia, lactic acid and seven carboxylic acids. The inhibitory effect was much less when the dose was reduced and applied in a blend with 6 compounds. The behavioral effect of 3-methylbutanoic acid needs to be further examined. A remarkable species-specific difference was revealed when hexanoic acid was found to act as an inhibitor or to be ineffective, whereas in the anthropophilic Ae. aegypti it is an important kairomone. Semi-field and field studies are needed to confirm that the qualitative and quantitative composition of attractive blends for $A n$. gambiae reported here can be successfully employed in trapping strategies under field conditions.

\footnotetext{
Acknowledgements We thank Dr. F. Griepink (Plant Research International, Wageningen University and Research Centre, Wageningen, The Netherlands) and the professors Dr. D. Hall and Dr. S. Torr (Natural Resources Institute, University of Greenwich, Chatham Maritime, UK) for their advice on the use of LDPE sachets and for the materials provided. We are also thankful to F. van Aggelen, A. Gidding, and L. Koopman (Laboratory of Entomology, Wageningen University, Wageningen, The Netherlands) for rearing the mosquitoes. Dr. M. Posthumus (Laboratory of
}

Organic Chemistry, Natural Products Chemistry Group, Wageningen University, Wageningen, The Netherlands) is acknowledged for providing estimations of vapor pressures for various test compounds. We also thank G. Leenders (PSG Facility Services, Wageningen University and Research Centre, Wageningen, The Netherlands) for making adjustments to the olfactometer and trapping devices and Dr. H Smid (Laboratory of Entomology, Wageningen University, Wageningen, The Netherlands) for drawing Fig. 1. This study was financed by the Technology Foundation of The Netherlands Organisation for Scientific Research (STW-NWO, grant WBI.4834) and in part by a grant from the Foundation for the National Institutes of Health (NIH) through the Grand Challenges in Global Health Initiative (GCGH\#121).

Open Access This article is distributed under the terms of the Creative Commons Attribution Noncommercial License which permits any noncommercial use, distribution, and reproduction in any medium, provided the original author(s) and source are credited.

\section{References}

Acree, F., Jr., Turner, R. B., Gouck, H. K., BerozA, M., and SMITH, N. 1968. L-Lactic Acid: A mosquito attractant isolated from humans. Science 161:1346-1347.

Allan, S. A., Bernier, U. R., and Kline, D. L. 2006. Attraction of mosquitoes to volatiles associated with blood. J. Vector Ecol. 31:71-78.

Bernier, U. R., Kline, D. L., BARnard, D. R., Schreck, C. E., and Yost, R. A. 2000. Analysis of Human Skin Emanations by Gas Chromatography/Mass Spectrometry. 2. Identification of volatile compounds that are candidate attractants for the yellow fever mosquito (Aedes aegypti). Anal. Chem. 72:747-756.

Bernier, U. R., Kline, D. L., Schreck, C. E., Yost, R. A., and BARNARD, D. R. 2002. Chemical analysis of human skin emanations: comparison of volatiles from humans that differ in attraction of Aedes aegypti (Diptera: Culicidae). J. Am. Mosq. Control Assoc. 18:186-195.

Bosch, O. J., GeIER, M., and BOECKH, J. 2000. Contribution of fatty acids to olfactory host finding of female Aedes aegypti. Chem. Senses 25:323-330.

BraKs, M. A. H., MeiJerinK, J., and TaKken, W. 2001. The response of the malaria mosquito, Anopheles gambiae, to two components of human sweat, ammonia and L-lactic acid, in an olfactometer. Physiol. Entomol. 26:142-148.

Curran, A. M., Rabin, S. I., Prada, P. A., and Furton, K. G. 2005. Comparison of the volatile organic compounds present in human odor using SPME-GC/MS. J. Chem. Ecol. 31:1607-1619.

DE JonG, R., and KNOLs, B. G. J. 1995. Selection of biting sites on man by two malaria mosquito species. Cell. Mol. Life Sci. 51:80-84.

DE Jong, R and KnOls, B. G. J. 1996. Selection of biting sites by mosquitoes, pp. 89-103, in G. R. Bock and G. Cardew (eds.). Olfaction in Mosquito Host Interactions. Wiley, Chichester.

DekKer, T., SteiB, B., CARdé, R. T., and Geier, M. 2002. 1-Lactic acid, a human-signifying host cue for the anthropophilic mosquito Anopheles gambiae sensu stricto. Med. Vet. Entomol. 16:91-98.

EIRAS, A. E., and JePson, P. C. 1991. Host location by Aedes aegypti (Diptera Culicidae): a wind tunnel study of chemical cues. Bull. Entomol. Res. 81:151-160.

Gallagher, M., Wysocki, C. J., Leyden, J. J., Spielman, A. I., Sun, X., and PretI, G. 2008. Analyses of volatile organic compounds from human skin. Br. J. Dermatol. 159:780-791.

GEIER, M., SASS, H., and BOECKH, J. 1996. A search for components in human body odour that attract female Aedes aegypti, pp. 132148, in G. R. Bock and G. Cardew (eds.). Olfaction in Mosquito Host Interactions. Wiley, Chichester. 
Geier, M., Bosch, O. J., and BoeckH, J. 1999. Ammonia as an attractive component of host odour for the Yellow fever mosquito Aedes aegypti. Chem. Senses 24:647-653.

HadDow, A. J., and SsenKubUge, Y. 1973. The mosquito of bwamba county, Uganda. IX. Further studies on the biting behaviour of an outdoor population of the Anopheles gambiae Giles complex. Bull. Ent. Res. 62:407-414.

Healy, T. P., and Copland, M. J. W. 2000. Human sweat and 2-oxopentanoic acid elicid a landing response from Anopheles gambiae. Med. Vet. Entomol. 14:195-200.

James, A. G., CASEy, J., Hyliands, D., and Mycock, G. 2004 Fatty acid metabolism by cutaneous bacteria and its role in axillary malodour. World J. Microbiol. Biotech. 20:787-793.

Killeen, G., Kihonda, J., Lyimo, E., OKetch, F., Kotas, M., Mathenge, E., Schellenberg, J., Lengeler, C., Smith, T., and DRAKELEY, C. 2006. Quantifying behavioural interactions between humans and mosquitoes: Evaluating the protective efficacy of insecticidal nets against malaria transmission in rural Tanzania. BMC Infect. Dis. 6:161.

KuINE, D. L. 1999. Comparison of two American biophysics mosquito traps: the professional and a new counterflow geometry trap. $J$. Am. Mosq. Control Assoc. 15:276-282.

Knols, B. G. J., VAn Loon, J. J. A., Cork, A., Robinson, R. D., Adam, W., Meijerink, J., De Jong, R., and TaKken, W. 1997. Behavioural and electrophysiological responses of the female malaria mosquito Anopheles gambiae (Diptera: Culicidae) to Limburger cheese volatiles. Bull. Ent. Res. 87:151-159.

KröcKel, U., Rose, A., EIRAS, A. E., and GEIER, M. 2006. New tools for surveillance of adult yellow fever mosquitoes: comparison of trap catches with human landing rates in an urban environment. J. Am. Mosq. Control Assoc. 22:229-238.

Maxwell, C. A., Wakibara, J., Tho, S., and Curtis, C. F. 1998. Malaria-infective biting at different hours of the night. Med. Vet. Entomol. 12:325-327.

Mboera, L. E. G., Knols, B. G. J., TAKKen, W., and Huisman, P. W. T. 1998. Olfactory responses of female Culex quinquefasciatus Say (Diptera: Culicidae) in a dual-choice olfactometer. J. Vector Ecol. 23:107-113.

MeijerinK, J., BraKs, M. A. H., Brack, A. A., AdAM, W., DeKKER, T., Posthumus, M. A., Van Beek, T. A., and Van LoOn, J. J. A. 2000. Identification of Olfactory Stimulants for Anopheles gambiae from Human Sweat Samples. J. Chem. Ecol. 26:1367-1382.

Penn, D. J., Oberzaucher, E., Grammer, K., Fischer, G., Soini, H. A., Wiesler, D., Novotny, M. V., DiXon, S. J., XU, Y., and BRERETON, R. G. 2007. Individual and gender fingerprints in human body odour. J. R. Soc. Interface 4:331-340.

Puri, S. N., MendKi, M. J., Sukumaran, D., Ganesan, K., Prakash, S., and SEKHAR, K. 2006. Electroantennogram and behavioral responses of Culex quinquefasciatus (Diptera :
Culicidae) females to chemicals found in human skin emanations. J. Med. Entomol. 43:207-213.

Qiu, Y. T., Smallegange, R. C., Hoppe, S., Van Loon, J. J. A., BAKKER, E. -J., and TAKKEN, W. 2004. Behavioural and electrophysiological responses of the malaria mosquito Anopheles gambiae Giles sensu stricto (Diptera: Culicidae) to human skin emanations. Med. Vet. Entomol. 18:429-438.

Qiu, Y. T., Smallegange, R. C., Van LoOn, J. J. A., Ter BraAK, C. J. F., and TAKKEN, W. 2006a. Interindividual variation in the attractiveness of human odours to the malaria mosquito Anopheles gambiae s. s. Med. Vet. Entomol. 20:280-287.

QiU, Y. T., VAN LoON, J. J. A., TAKKen, W., MeIJerink, J., and Smid, H. M. 2006b. Olfactory coding in antennal neurons of the malaria mosquito, Anopheles gambiae. Chem. Senses 31:845-863.

Schreck, C. E., Smith, N., Carlson, D. A., Price, G. D., Haile, D., and GoDwIN, D. R. 1981. A material isolated from human hands that attracts female mosquitoes. J. Chem. Ecol. 2:429-438.

SMALlEGANGe, R. C., GEIER, M., and TAKKEN, W. 2002. Behavioural responses of Anopheles gambiae to ammonia, lactic acid and a fatty acid in a y-tube olfactometer, pp. 147-152. Proc. Exp. Appl. Entomol., Vol. 13, N.E.V., Amsterdam.

Smallegange, R. C., Qiu, Y. T., Van Loon, J. J. A., and TAKKen, W. 2005. Synergism between ammonia, lactic acid and carboxylic acids as kairomones in the host-seeking behaviour of the malaria mosquito Anopheles gambiae sensu stricto (Diptera: Culicidae). Chem. Senses 30:145-152.

Smith, C. N., Smith, N., Gouck, H. K., WeidhaAs, D. E., Gilbert, I. H., MAYER, M. S., SMitTle, B. J., and HofBAuer, A. 1970. LLactic acid as a factor in the attraction of Aedes aegypti (Diptera: Culicidae) to human hosts. Ann. Entomol. Soc. Am. 63:760-770.

Torr, S. J., Hall, D. R., Phelps, R. J., and Vale, G. A. 1997. Methods for dispensing odour attractants for tsetse flies (Diptera: Glossinidae). Bull. Ent. Res. 87:299-311.

VALE, G. A., and HALL, D. R. 1985. The use of 1-octen-3-ol, acetone and carbon dioxide to improve baits for tsetse flies, Glossina spp. (Diptera: Glossinidae). Bull. Ent. Res. 75:219-231.

Williams, C. R., Bergbauer, R., Geier, M., Kline, D. L., Bernier, U. R., Russell, R. C., and RITCHIE, S. A. 2006a. Laboratory and field assessment of some kairomone blends for host-seeking Aedes aegypti. J. Am. Mosq. Control Assoc. 22:641-647.

Williams, C. R., Long, S. A., Russell, R. C., and Ritchie, S. A. 2006b. Field efficacy of the BG-Sentinel compared with CDC Backpack Aspirators and CO2-baited EVS traps for collection of adult Aedes aegypti in Cairns, Queensland, Australia. J. Am. Mosq. Control Assoc. 22:296-300.

Williams, C. R., Ritchie, S. A., Russell, R. C., Eiras, A. E., Kline, D. L., and GEIER, M. 2006c. Geographic variation in attraction to human odor compounds by Aedes aegypti mosquitoes (Diptera: Culicidae): a laboratory study. J. Chem. Ecol. 32:1625-1634. 